\title{
Dexmedetomidine preconditioning protects against lung injury induced by ischemia-reperfusion through inhibition of autophagy
}

\author{
WEI ZHANG and JIAQIANG ZHANG
}

\begin{abstract}
Department of Anesthesiology, Henan Provincial People's Hospital, Zhengzhou, Henan 450003, P.R. China
\end{abstract}
Received April 25, 2016; Accepted March 24, 2017

DOI: $10.3892 /$ etm.2017.4623

\begin{abstract}
The present study aimed to evaluate the role of autophagy in the protective effect of dexmedetomidine in lung injury caused by ischemia-reperfusion (IR) in rats. In total 48 adult male Sprague-Dawley rats were randomly divided into 6 groups ( $=8$ ) as follows: i) Sham group; ii) the IR group; iii) IR $+1 \mu \mathrm{g} / \mathrm{kg}$ dexmedetomidine preconditioning group (pre-LD); iv) $\mathrm{IR}+10 \mu \mathrm{g} / \mathrm{kg}$ dexmedetomidine preconditioning group (pre-HD); v) IR $+1 \mu \mathrm{g} / \mathrm{kg}$ dexmedetomidine postconditioning group (post-LD); and vi) IR $+10 \mu \mathrm{g} / \mathrm{kg}$ dexmedetomidine postconditioning group (post-HD). After the rats were anesthetized, the hilum of the left lung was occluded with a non-invasive microvascular clip for $30 \mathrm{~min}$ to induce ischemia. The clip was then removed and the left lung was allowed to regain ventilation and blood for $2 \mathrm{~h}$. The rats were then sacrificed, the left lung removed and the wet/dry (W/D) lung weight ratio was determined. Pathological changes to the lungs were evaluated by light and transmission electron microscopy. Furthermore, the rate of lung cell apoptosis was determined by the TUNEL assay. The expression of hypoxia-inducible factor $1 \alpha$ (HIF-1 $\alpha)$, Bcl-2/adenovirus E1B 19-kDa interacting protein 3 (BNIP3), BNIP3 like (BNIP3 L) and microtubule-associated protein 1A/1B light chain 3B (LC3II) was determined by western blotting. Compared with the sham group, a significant increase in the W/D lung weight ratio, and malondialdehyde (MDA), BNIP3, BNIP3 L and LC3II levels were observed in the IR group, and HIF-1 $\alpha$ levels and superoxide dismutase (SOD) activity were decreased. Furthermore, the W/D ratio was lower in the pre-LD and pre-HD groups than in the IR group. Additionally, SOD activity was significantly higher and MDA expression was significantly lower in the pre-LD and pre-HD groups compared with the IR group. BNIP3, BNIP3 L and LC3II protein levels were significantly lower in the pre-LD and pre-HD groups compared with the IR group, while HIF-1 $\alpha$
\end{abstract}

Correspondence to: Dr Jiaqiang Zhang, Department of Anesthesiology, Henan Provincial People's Hospital, 7 Wei Wu Road, Zhengzhou, Henan 450003, P.R. China

E-mail: mazuizhang@163.com

Key words: autophagy, apoptosis, dexmedetomidine, ischemicreperfusion, lung injury was notably upregulated in the pre-LD and pre-HD groups compared with the IR group. In conclusion, the results of the present study indicate that dexmedetomidine preconditioning protects against lung injury induced by IR through inhibition of autophagy and apoptosis.

\section{Introduction}

Lung ischemia-reperfusion injury (LIRI), a form of acute sterile lung injury, remains a frequent complication that may result in morbidity and mortality during lung transplantation (1), cardiopulmonary bypass (2), trauma (3), pulmonary embolism (4) and resuscitation in hemorrhagic shock (5). Previous studies have demonstrated that dexmedetomidine may protect against lung injury by promoting the expression of heme oxygenase-1 (6). Pre-administration of dexmedetomidine prior to ischemia-reperfusion (IR) has been identified to reduce pulmonary damage and decrease myeloperoxidase activation in lung tissue, in addition to levels of proinflammatory cytokines in the bronchoalveolar lavage fluid (7). The results of this previous study indicated that dexmedetomidine exerted these effects through the toll-like receptor 4/myeloid differentiation primary response $88 /$ mitogen-activated protein kinase signaling pathway (7).

Autophagy is a cell protective mechanism that is activated in response to stress signals from the endoplasmic reticulum (8). Autophagy occurs from the embryonic period (9) and is fundamental for the growth and differentiation of tissues. Autophagy is involved in the growth and differentiation of alveolar cells $(10,11)$. Depletion of energy and an oxygen deficient environment are triggers for autophagy. It is well known that autophagic cellular damage during ischemia and reperfusion is the basis of ischemia-reperfusion injury (IRI). This is an attempt to survive the severely limiting conditions during ischemia. Previous studies have investigated the role of autophagy in IRI. However, it remains controversial whether high levels of autophagy lessen or aggravate IRI $(12,13)$. Autophagy occurs at low levels basally to mediate homeostatic functions, including organelle and protein turnover. Furthermore, autophagy increases when intracellular nutrition and energy are deficient; for example, in hypoxia or starvation. Autophagy can also trigger apoptosis (14).

A previous study demonstrated that ischemia and immediate reperfusion can trigger autophagy, and that autophagy participates in renal IRI (12). Furthermore, autophagy serves 
a protective role in LIRI (15). However, in a previous study indicated that autophagy was activated and served a role in the pathophysiological process of LIRI (16). Whether autophagy is associated with the protective effect of dexmedetomidine in LIRI remains unclear. The present study aimed to investigate the underlying molecular mechanism of the protective effect of dexmedetomidine in LIRI.

\section{Materials and methods}

Animals. The present study was approved by the Animal Care and Use Committee of Henan Provincial People's Hospital (Zhengzhou, China). Adult (aged 8-10 weeks) male Sprague-Dawley rats ( $\mathrm{n}=48$; weight, 250-300 g) were provided by Zhengzhou University Laboratory Animal Center (Zhengzhou, China). The animals were housed in plastic cages with water and food available ad libitum. The temperature $\left(22 \pm 1^{\circ} \mathrm{C}\right)$ and humidity $(60 \pm 5 \%)$ were controlled in a room with a 12 -h light-dark cycle. All the rats were acclimatized to these conditions for 1 week prior to the study.

Establishment of LIRI models. All rats were anesthetized by intraperitoneal injection of $400 \mathrm{mg} / \mathrm{kg} 10 \%$ chloral hydrate (Batch no. 20150121; Tianjin Guangfu Fine Chemical Research Institution, Tianjin, China), and the caudal vein was cannulated for fluid and drug administration. After tracheostomy was completed, all rats were endotracheally intubated with a vein puncture needle and then connected to a breathing machine (Model: HX-100E; Temo Technology Co., Ltd, Beijing, China) specialized for small animals. Rats were placed in a right lateral position and the left lung was exposed through the fifth intercostal space. The hilum of the left lung was occluded with a non-invasive microvascular clip for $30 \mathrm{~min}$. The clip was removed at the end of the ischemic period, and the left lung was allowed to regain ventilation and blood for $2 \mathrm{~h}$. After the experiment was finished, the rats were sacrificed via exsanguination by cardiac puncture and the left lung was removed for further analysis.

Experimental protocol and drug administration. A total of 48 rats were randomly allocated into six groups ( $\mathrm{n}=8$ per group) as follows: i) The sham group received saline administration ( $1 \mathrm{ml} / \mathrm{h}$ intravenously) without LIRI; ii) the IR group received saline administration ( $1 \mathrm{ml} / \mathrm{h}$ intravenously) following LIRI; iii) the LIRI $+1 \mu \mathrm{g} / \mathrm{kg}$ dexmedetomidine preconditioning group [pre-low dose (LD)] received $1 \mu \mathrm{g} / \mathrm{kg}$ dexmedetomidine prior to LIRI; iv) the LIRI $+10 \mu \mathrm{g} / \mathrm{kg}$ dexmedetomidine preconditioning group [pre-high dose (HD)] received $10 \mu \mathrm{g} / \mathrm{kg}$ dexmedetomidine prior to LIRI; v) the IR $+1 \mu \mathrm{g} / \mathrm{kg}$ dexmedetomidine postconditioning group (post-LD) received $1 \mu \mathrm{g} / \mathrm{kg}$ dexmedetomidine following LIRI; and vi) the IR +1 $0 \mu \mathrm{g} / \mathrm{kg}$ dexmedetomidine postconditioning group (post-HD) received $10 \mu \mathrm{g} / \mathrm{kg}$ dexmedetomidine following LIR.

Drug administration. Dexmedetomidine (Batch no. 15030932; Hengrui Medicine Co., Ltd., Lianyungang, China) preconditioning [0.1 (pre-LD) or 1 (pre-HD) $\mu \mathrm{g} / \mathrm{kg} / \mathrm{min}$ total over $10 \mathrm{~min}]$ was administered intravenously after the caudal vein was opened. Dexmedetomidine postconditioning [0.1 (post-LD) or 1 (post-HD) $\mu \mathrm{g} / \mathrm{kg} / \mathrm{min}$ total over $10 \mathrm{~min}$ ) was administered immediately after the reperfusion period started.

Wet/dry (W/D) lung weight ratio. The W/D weight ratio for 3 rats from each group was calculated. The upper lobe of the left lung was immediately weighed following harvest to obtain the wet weight and again following desiccation in a $60^{\circ} \mathrm{C}$ oven for $48 \mathrm{~h}$ to obtain the dry weight. The lung water content was then assessed by the W/D ratio.

Examination of lung injury by light microscopy. The left lungs from 2 rats from each group were perfused with $4 \%$ formaldehyde (room temperature for $24 \mathrm{~h}$ ). The formaldehyde infused left lung samples were embedded in paraffin wax $\left(58^{\circ} \mathrm{C}\right.$ for $2 \mathrm{~h}$ ), sectioned (5 $\mu \mathrm{m}$ thick) and stained with hematoxylin and eosin. The samples were subsequently examined using light microscopy at a magnification of x200 and the mean value of three fields of view was used as to assess the lung injury.

Examination of lung injury by transmission electron microscopy. The left lungs from 2 rats from each group were perfused with $2.5 \%$ glutaraldehyde solution (precooled at $4^{\circ} \mathrm{C}$ ) and preserved. Following fixation at $4^{\circ} \mathrm{C}$ for $24 \mathrm{~h}$ with $2.5 \%$ glutaraldehyde, the samples were treated with $1 / 15 \mathrm{M}$ phosphate buffer for $10-15 \mathrm{~min}$, followed by $50,70,80,70$ and $100 \%$ acetone dehydration washes for 10-15 min each (at room temperature). Tissues were then embedded with EPON 812 resin (at $60^{\circ} \mathrm{C}$ for $48 \mathrm{~h}$ ), sectioned into 50-nm-thick ultrathin slices, stained with citric acid and uranyl acetate (room temperature for $30 \mathrm{~min}$ ) and images captured by transmission electron microscopy (H-7500; Hitachi, Tokyo, Japan). The mean value of three fields of view were used to assess the lung injury.

Superoxide dismutase (SOD) activity and malondialdehyde (MDA) concentration. An ultraviolet spectrophotometer was used to perform a colorimetric determination at a wavelength of $550 \mathrm{~nm}$. Lipid peroxidation was determined by measuring the rate of production of thiobarbituric acid reactive substances (expressed as MDA equivalents) Lung tissues were added to precooled PBS $\left(4^{\circ} \mathrm{C}\right)$ and then centrifuged $\left(1,006.2 \times\right.$ g at $4^{\circ} \mathrm{C}$ for $15 \mathrm{sec}$ ) into tissue homogenates by a low-speed homogenate slurry machine (30 sec per time, interval ice bath for $1 \mathrm{~min}$ ). Following centrifugation by the aforementioned methods, the tissue homogenates were first placed under a $37^{\circ} \mathrm{C}$ water bath for $40 \mathrm{~min}$ and then placed at room temperature for $10 \mathrm{~min}$. The activity of SOD and the concentration of MDA were determined by a $550 \mathrm{~nm}$ colorimetric assay according to the manufacturer's protocol (SOD cat no: A001-1 and MDA cat no: A003-1; Nanjing Jiancheng Bioengineering Institute, Nanjing, China).

Detection of lung cell apoptosis by the TUNEL assay. The TUNEL assay was employed according to manufacturer's protocol of the In situ cell death detection kit-POD (cat no. 11684817910; Roche, Basel, Switzerland). Apoptotic cells were indicated by brown-yellow granules in the cytoplasm. The number of apoptotic cells in five random fields of view (magnification, $\mathrm{x} 400$ ) was calculated. The apoptosis index (AI; \%) was expressed as follows: The number of apoptotic cells/ the total number of cells x100. 


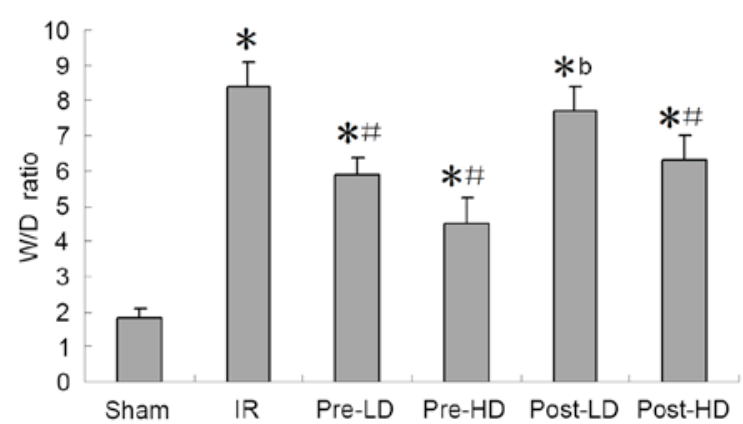

Figure 1. W/D lung weight ratio results following IR injury. "P<0.05 vs. the sham group; ${ }^{\text {}} \mathrm{P}<0.05$ vs. the IR group; ${ }^{\mathrm{P}} \mathrm{P}<0.05$ vs. the pre-HD group. W/D, wet/dry; IR, ischemia reperfusion; pre-LD, low-dose dexmedetomidine preconditioning; pre-HD, high-dose dexmedetomidine preconditioning; post-LD, low-dose dexmedetomidine postconditioning; post-HD, high-dose dexmedetomidine postconditioning.

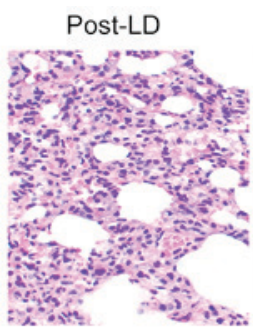

Post-HD
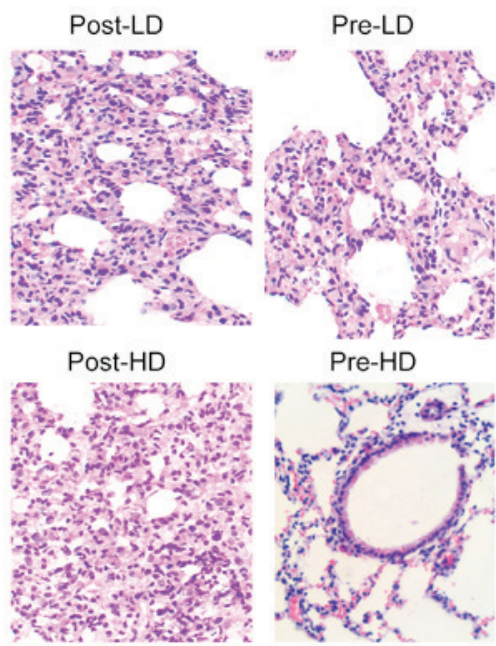

Pre-HD
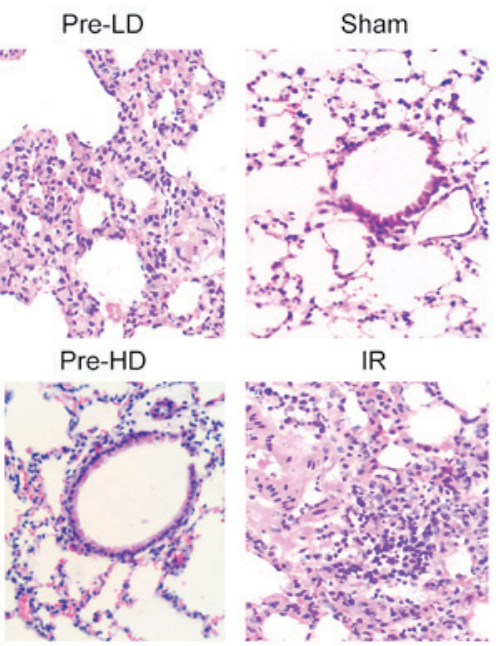

IR

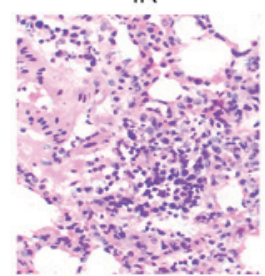

Figure 2. Examination of lung injury by light microscopy following IR injury. The histological analysis revealed minimal lung injury in the sham group, while severe lung injury was observed in the IR group, in which pulmonary edema, inflammatory cell infiltration and alveolar congestion were evident Furthermore, injuries in the pre-HD group were mild. Magnification x 200 . IR, ischemia reperfusion; pre-LD, low-dose dexmedetomidine preconditioning; pre-HD, high-dose dexmedetomidine preconditioning; post-LD, low-dose dexmedetomidine postconditioning; post-HD, high-dose dexmedetomidine postconditioning.

Western blotting. The lower lobes of the left lungs of 4 rats from each group were used for western blotting $(n=24,4$ from each group). The lung tissues were mixed with RIPA lysis buffer (cat no. P0013; Beyotime Institute of Biotechnology, Shanghai, China) and centrifuged at $10,000 \mathrm{x} \mathrm{g}$ at $4^{\circ} \mathrm{C}$ for $10 \mathrm{~min}$. According to the manufacturer's protocol, protein quantities in the liquid supernatant were detected using the bicinchoninic acid method (Enhanced BCA Protein Assay kit; cat no, P0009; Beyotime Institute of Biotechnology). Then, 12\% SDS-PAGE electrophoresis was performed using 80 ug of protein loaded per lane) and proteins were transferred onto a nitrocellulose membrane. The membranes were blocked with 5\% skimmed milk in Tris-buffered saline with Tween 20 for $1 \mathrm{~h}$ at room temperature, and then incubated overnight at $4^{\circ} \mathrm{C}$ with rabbit polyclonal antibodies directed against hypoxia-inducible factor $1 \alpha$ (HIF-1 $\alpha$; cat no. sc13515; 1:1,000; Santa Cruz Biotechnology, Inc., Dallas, TX, USA),
Bcl-2/adenovirus E1B 19-kDa interacting protein 3 (BNIP3; cat no. sc56167; 1:1,000; Santa Cruz Biotechnology, Inc.), BNIP3-like (BNIP3L; cat no. ab109414; 1:1,000; Abcam, Cambridge, USA) and microtubule-associated protein 1A/1B light chain 3B (LC3II; cat no. sc271625; 1:1,000; Santa Cruz Biotechnology Inc.). After washing, rabbit polyclonal antibodies (secondary antibodies: HRP-labeled rabbit Anti-Goat IgG (H+L), cat no. ZB-2306, 1:5,000; HRP-labeled Goat Anti-Mouse IgG (H+L), cat no. ZB-2305, 1:2,500; ZSGB-BIO, Beijing, China) directed against BNIP3, BNIP3 L, LC3II and HIF-1 $\alpha$ were applied to the membranes for $1 \mathrm{~h}$ at room temperature, and GAPDH rabbit polyclonal antibody (cat no. sc 47724; 1:1,200; Santa Cruz Biotechnology, Inc.) was used as a control. According to the manufacturer's protocol, Protein bands were then visualized using enhanced chemiluminescence detection reagents (BeyoECL Plus; cat no. P0018; Beyotime Institute of Biotechnology), equal volume of liquid A and B were mixed and once coated to the membrane, they were exposed to photographic film. The images were then analyzed and protein expression quantified using Image J2x (version 2.1.4.7) software (National Institutes of Health, Bethesda, MD, USA).

Statistical analysis. All data are presented as the mean \pm standard deviation. All statistical tests were performed using SPSS software (version 17.0; SPSS, Inc., Chicago, IL, USA). The statistical differences between groups were assessed by one-way analysis of variance followed by Bonferroni correction. $\mathrm{P}<0.05$ was considered to indicate a statistically significant difference.

\section{Results}

Preconditioning with $H D$ dexmedetomidine reduces the W/D ratio following LIRI. The W/D ratios of the IR and post-LD groups were significantly higher compared with the sham group $(\mathrm{P}<0.05$; Fig. 1). The W/D ratio of the post-LD group was also significantly higher compared with that of the pre-HD group $(\mathrm{P}<0.05$; Fig. 1$)$. This indicates that preconditioning with a $\mathrm{HD}$ of dexmedetomidine significantly reduces the W/D ratio following LIRI.

Preconditioning with HDdexmedetomidinereduces lung injury following LIRI. Lung injury was evaluated by the standards as follows (light microscopy): Pulmonary interstitial edema, alveolar edema, alveolar congestion and neutrophil infiltration. As illustrated in Fig. 2, histological analysis revealed minimal lung injury in the sham group, while severe lung injury was identified in the IR group and post-LD group. Furthermore, no notable difference in lung injury was revealed between the pre-LD and post-HD groups. By contrast, the lung tissues harvested from the rats in the pre-HD group exhibited notably milder injuries compared with the IR, pre-LD, post-LD and post-HD groups. As presented in Fig. 3, lung injury was assessed through electron microscopy and similar to the results from light microscopy, no significant injury was observed in the sham group, but a significant decrease of alveolar type II epithelium villi and lamellar corpuscles were observed in the IR group. Preconditioning with dexmedetomidine attenuated these injuries. 

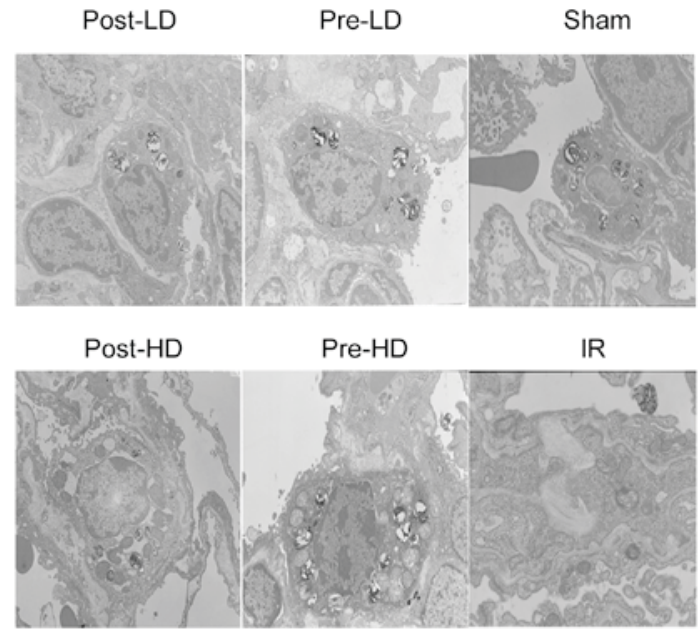

Figure 3. Examination of lung injury by transmission electron microscopy following IR injury. Similar to the results from light microscopy, no significant injury was observed in the sham group, but a significant decrease of alveolar type II epithelium villi and lamellar corpuscles were observed in the IR group. Preconditioning with dexmedetomidine attenuated these injuries. Magnification, x3,000. IR, ischemia-reperfusion; pre-LD, low-dose dexmedetomidine preconditioning; pre-HD, high-dose dexmedetomidine preconditioning; post-LD, low-dose dexmedetomidine postconditioning; post-HD, high-dose dexmedetomidine postconditioning.

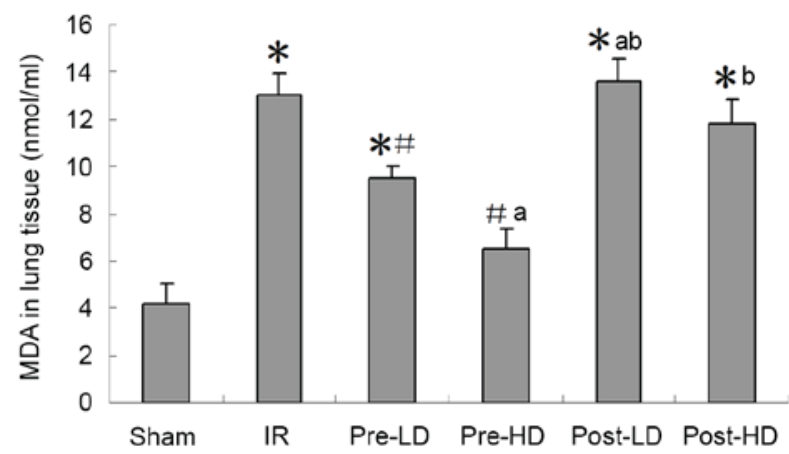

Figure 4. MDA concentration in lung tissue following IR injury. ${ }^{*}<0.05$ vs. the sham group; ${ }^{\#} \mathrm{P}<0.05$ vs. the IR group; ${ }^{\mathrm{a}} \mathrm{P}<0.05$ vs. the pre-LD group; ${ }^{\mathrm{b}} \mathrm{P}<0.05$ vs. the pre-HD group. MDA, malondialdehyde; IR, ischemia-reperfusion; pre-LD, low-dose dexmedetomidine preconditioning; pre-HD, high-dose dexmedetomidine preconditioning; post-LD, low-dose dexmedetomidine postconditioning; post-HD, high-dose dexmedetomidine postconditioning.

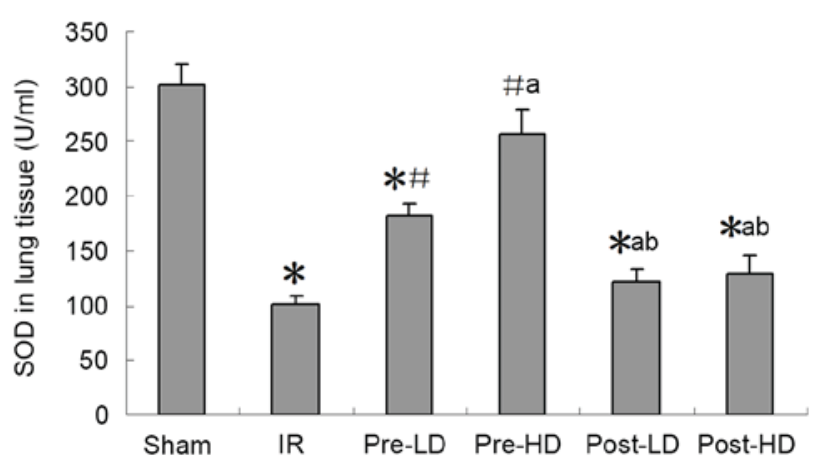

Figure 5. SOD activity in lung tissue following IR injury. ${ }^{*} \mathrm{P}<0.05$ vs. the sham group; ${ }^{\text {"}} \mathrm{P}<0.05$ vs. the IR group; ${ }^{\mathrm{a}} \mathrm{P}<0.05$ vs. the pre-LD group; ${ }^{\text {b }} \mathrm{P}<0.05$ vs. the pre-HD group. SOD, superoxide dismutase; IR, ischemia-reperfusion; pre-LD, low-dose dexmedetomidine preconditioning; pre-HD, high-dose dexmedetomidine preconditioning; post-LD, low-dose dexmedetomidine postconditioning; post-HD, high-dose dexmedetomidine postconditioning.
Preconditioning with $H D$ and $L D$ dexmedetomidine reduces lower MDA and higher SOD following LIRI SOD activity and $M D A$ expression. As presented in Figs. 4 and 5, a significantly higher MDA level and significantly lower SOD activity in the left lung were observed in the IR group compared with the sham group (both $<0.05$ ). Compared with the IR group, higher SOD and lower MDA were observed in the pre-LD and pre-HD groups (all $\mathrm{P}<0.05$ ). However, no significant differences in MDA levels of SOD activity were observed in the post-LD and post-HD groups compared with the IR group.

Preconditioning with $H D$ dexmedetomidine reduces AI following LIRI. As demonstrated in Figs. 6 and 7, compared with the sham group, the AI index of the other five groups were significantly increased $(\mathrm{P}<0.05)$. However no significant difference in AI was observed between the IR and post-LD groups. Furthermore, compared with the IR group, the AI index of the pre- $\mathrm{LD}$, pre-HD and post-HD groups was significantly lower (all $\mathrm{P}<0.05$ ). In addition, no significant difference in $\mathrm{AI}$ was observed between the pre-LD and post-HD groups. However, the AI of the pre-HD group was significantly lower compared with the pre-LD group, while the post-LD and post-HD groups exhibited a significant increase in AI when compared with the pre-HD group ( $\mathrm{P}<0.05$; Fig. 6).

Preconditioning with $H D$ dexmedetomidine reduces the level of BNIP3, BNIP3L and LC3II. As illustrated in Figs. 8 and 9, expression of BNI3P, BNI3PL and LC3II protein was significantly higher in the IR, pre-LD, post-LD and post-HD groups compared with the sham group (all $\mathrm{P}<0.05$ ). Compared with the IR group, BNIP3, BNIP3 L and LC3II protein levels were significantly lower in the pre-LD and pre-HD groups (all $\mathrm{P}<0.05$ ). Notably, the levels of BNIP3, BNIP3 L and LC3II protein were lower in the pre-HD group compared with the pre-LD group, although this difference was not statistically significant. Furthermore, BNIP3, BNIP3 L and LC3II protein levels were significantly lower in the post-HD group compared with the IR group (all $\mathrm{P}<0.05$ ); however, no significant differences were observed between the pre-LD and post-HD groups.

Preconditioning with $H D$ dexmedetomidine reduces the level of HIF-1 $\alpha$. The level of HIF-1 $\alpha$ protein was notably lower in the IR compared with the sham group, although this difference was not statistically significant (Figs. 8 and 9). In addition, compared with the IR group, HIF-1 $\alpha$ protein levels were higher in the pre-LD and pre-HD groups. There was not marked differences in HIF-1 $\alpha$ levels between the post-LD, post-HD and IR groups.

\section{Discussion}

In the present study, lung damage was evaluated by determination of the W/D ratio and imaging techniques. High water content in the lung is a representative symptom of acute lung injury (17), as demonstrated by the severe lung damage observed in the IR group. The results of the present study demonstrated that LD and HD preconditioning with dexmedetomidine attenuated the development of pulmonary edema, as indicated by the significant decrease in the lung W/D ratio compared with the IR group. However, compared with the IR group, 


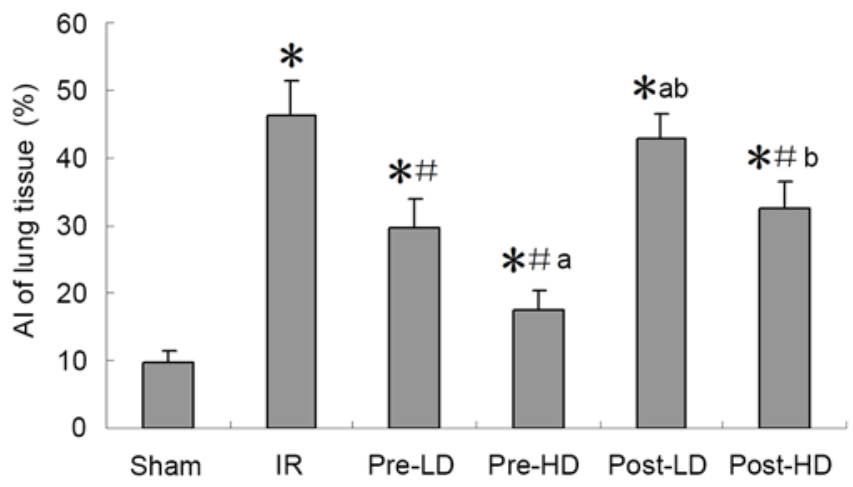

Figure 6. AI index of lung tissue following IR injury. The sham group had the lowest AI, while the IR group had the highest AI. Furthermore, pre-HD significantly decreased the AI. ${ }^{*} \mathrm{P}<0.05$ vs. the sham group; ${ }^{\#} \mathrm{P}<0.05$ vs. the IR group; ${ }^{\mathrm{a}} \mathrm{P}<0.05$ vs. the pre-LD group; ${ }^{\mathrm{b}} \mathrm{P}<0.05$ vs. the pre-HD group. AI, apoptosis index; IR, ischemia-reperfusion; pre-LD, low-dose dexmedetomidine preconditioning; pre-HD, high-dose dexmedetomidine preconditioning; post-LD, low-dose dexmedetomidine postconditioning; post-HD, high-dose dexmedetomidine postconditioning.

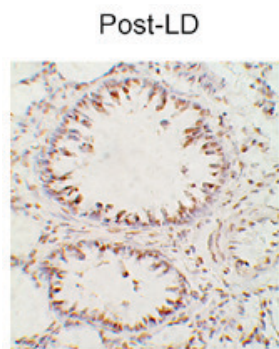

Post-HD

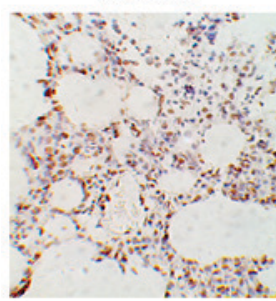

Pre-LD

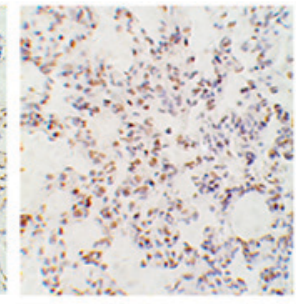

Pre-HD

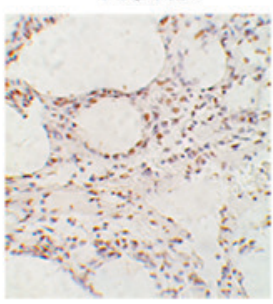

Sham

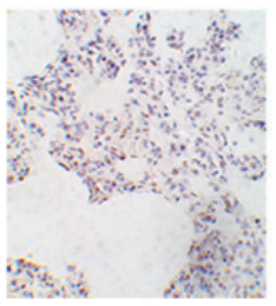

IR

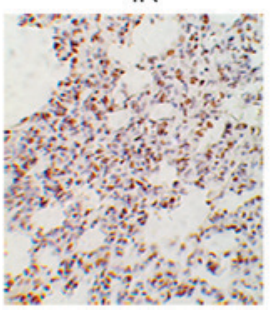

Figure 7. Representative images from the TUNEL apoptosis assay following IR injury. Yellow-brown staining indicates apoptosis. Magnification, x400. IR, ischemia-reperfusion; pre-LD, low-dose dexmedetomidine preconditioning; pre-HD, high-dose dexmedetomidine preconditioning; post-LD, low-dose dexmedetomidine postconditioning; post-HD, high-dose dexmedetomidine postconditioning.

a significance decrease in the lung W/D ratio was observed only in the post-HD groups and not the post-LD group. These results indicate that preconditioning with dexmedetomidine can effectively attenuate the development of pulmonary edema; however, only a HD of dexmedetomidine could exert a similar effect when applied in a postconditioning manner. HD dexmedetomidine, applied in a post-conditioning manner had a similar effect to a LD applied in a preconditioning manner. However, HD dexmedetomidine attenuated the development pulmonary edema more effectively when applied in a preconditioning manner compared with a postconditioning manner.

Lung damage observed by light and transmission electron microscopy was consistent with the results of the W/D ratio. The results indicated that preconditioning with dexmedetomidine produces protection against LIRI in a dose-dependent manner. A previous study demonstrated that the injuries induced by intestinal IR in rats improved with the administration of $5 \mu \mathrm{g} / \mathrm{kg} / \mathrm{h}$ dexmedetomidine compared with $2.5 \mu \mathrm{g} / \mathrm{kg} / \mathrm{h}$ dexmedetomidine prior to ischemia (18). Furthermore, another study demonstrated dexmedetomidine preconditioning, but not postconditioning or treatment with the peripheral $\alpha 2$-adrenergic receptor agonist fadolmidine, ameliorated kidney IRI and inflammatory responses (19). The results of the present study indicate that HD dexmedetomidine postconditioning provides slight protection to the lungs, similar to LD dexmedetomidine applied in a preconditioning manner.

Reperfusion injury is directly associated with the formation of reactive oxygen species (ROS), endothelial cell injury, increased vascular permeability, and the activation and production of neutrophils, platelets, cytokines and the complement system (20). Furthermore, ROS serve a key role in the development of pulmonary injury, which is characterized by an increase in ROS and other free radicals, with an essential role in the sequence of events leading to lung failure (21). The importance of ROS in the pathophysiology of IRI was demonstrated by the injection of free radical scavengers or enzymes, including SOD, catalase and glutathione peroxidase, which was identified to prevent the damage that occurs during reperfusion $(22,23)$. In the present study, SOD activity in the lungs after IRI was significantly higher in the pre-LD and pre-HD groups compared with the IR group, indicating that pre-administration of dexmedetomidine effectively attenuates oxidative stress injuries induced by IR. Oxidative stress and lipid peroxidation serve an important role in distant organ damage following IR. Lipid peroxidation was determined by measuring the rate of production of thiobarbituric acid reactive substances (expressed as MDA equivalents) (24). In the present study, MDA levels were decreased by preconditioning with dexmedetomidine; however, postconditioning with dexmedetomine did not have a protective effect. The results of the present -study suggest that dexmedetomidine alleviates oxidative stress injury induced by IR, but only in a preconditioning manner.

Occlusion of the arterial blood supply is caused by an embolus, resulting in ischemia and consequently a serious imbalance between the supply and metabolic demand for oxygen, causing tissue hypoxia. The HIF-1 transcription factor, which is composed of oxygen-labile HIF-1 $\alpha$ and the constitutive HIF-1 $\beta$ subunit, is responsible for the response to hypoxia or ischemia. The activation of HIF-1 $\alpha$ serves a protective role in the IRI of several tissues through regulating inflammation (25). HIF-1 $\alpha$ contributes to pulmonary vascular dysfunction in LIRI and the HIF-1 $\alpha$ stabilizer dimethyloxalylglycine can attenuate IR-induced inhibition of endothelial function (26). In the present study, postconditioning with dexmedetomidine did not upregulate the expression of HIF-1 $\alpha$, while a marked upregulation of HIF-1 $\alpha$ was observed in the pre-LD and pre-HD groups.

Autophagy is a highly conserved process that occurs in all eukaryotes. The functional association between autophagy and cellular death or survival is complicated. Autophagy promotes cell survival under conditions of stress and starvation, whereas in other situations autophagic cell death may occur. Previous 


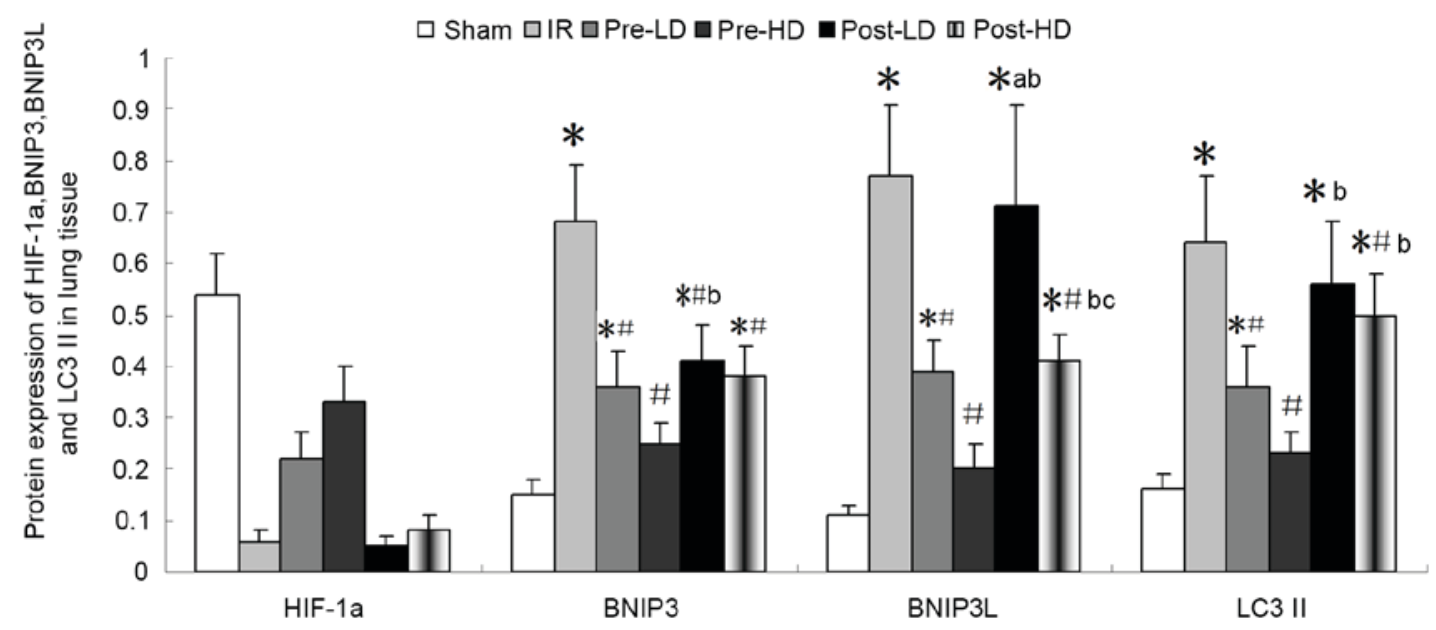

Figure 8. Quantitative western blot analysis of BNIP3, BNIP3 L, HIF-1 $\alpha$ and LC3II protein expression in lung tissue following IR injury. "P<0.05 vs. the sham group; ${ }^{\prime} \mathrm{P}<0.05$ vs. the IR group; ${ }^{b} \mathrm{P}<0.05$ vs. the pre-HD group; ${ }^{\mathrm{C}} \mathrm{P}<0.05$ vs. post-LD group. BNIP3, Bcl-2/adenovirus E1B $19-\mathrm{kDa}$ interacting protein 3 ; BNIP3 L, BNIP3 like; HIF-1 $\alpha$, hypoxia-inducible factor 1 $\alpha$; LC3II, microtubule-associated protein 1A/1B light chain 3B; IR, ischemia-reperfusion; pre-LD, low-dose dexmedetomidine preconditioning; pre-HD, high-dose dexmedetomidine preconditioning; post-LD, low-dose dexmedetomidine postconditioning; post-HD, high-dose dexmedetomidine postconditioning.

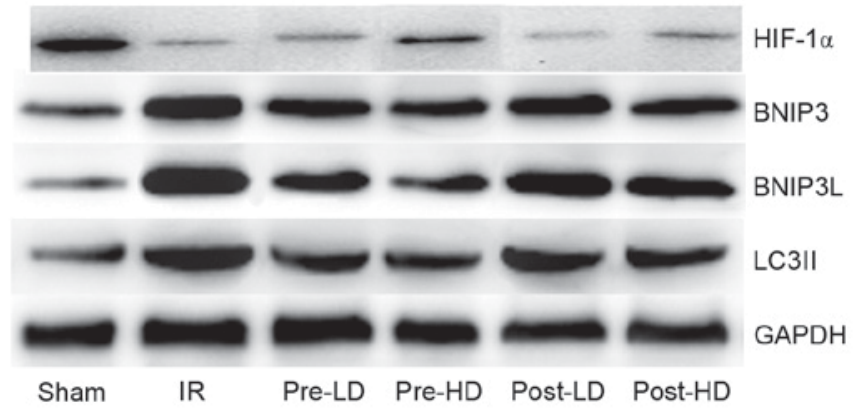

Figure 9. Western blots of BNIP3, BNIP3 L, HIF-1 $\alpha$ and LC3II. BNIP3, Bcl-2/adenovirus E1B 19-kDa interacting protein 3; BNIP3 L, BNIP3 like; HIF-1 $\alpha$, hypoxia-inducible factor $1 \alpha$; LC3II, microtubule-associated protein 1A/1B light chain 3B; IR, ischemia-reperfusion; pre-LD, low-dose dexmedetomidine preconditioning; pre-HD, high-dose dexmedetomidine preconditioning; post-LD, low-dose dexmedetomidine postconditioning; post-HD, high-dose dexmedetomidine postconditioning.

studies (27-29) have demonstrated that autophagy can be induced by various cellular conditions in IRI, including energy starvation, oxidative stress, endoplasmic reticulum stress and inflammation. Furthermore, the activation of autophagy may be a important process that enhances tissue tolerance to ischemia (30). The renoprotective effects of dexmedetomidine have been identified to be mediated partly by the maintenance of autophagy (21). It appears that autophagy serves a protective role in LIRI and that this protective effect is enhanced with increasing autophagy levels (31). However, it remains controversial whether high levels of autophagy lessen or increase IRI, particularly in lung tissue (16). As demonstrated in the present study, the expression of LC3II, a biomarker of autophagy, was upregulated by IR. Furthermore, preconditioning with dexmedetomidine can reduce autophagy levels; LC3II levels were significantly reduced by preconditioning with dexmedetomidine, particularly with HD dexmedetomidine.

Autophagy and apoptosis determine cellular fate. Autophagy and apoptosis are discrete cellular processes that are mediated by distinct groups of regulatory and executioner molecules $(32,33)$. The underlying mechanisms of the association between autophagy and apoptosis are not yet fully defined; however, recent investigations have revealed that several apoptotic proteins modulate autophagy $(34,35)$. The present study demonstrated that IR-induced apoptosis could be reduced by dexmedetomidine, particularly when administered in a preconditioning manner.

Bcl-2/adenovirus E1B 19-kDa interacting protein 3 (BNIP3) and $\mathrm{Bcl}-2 /$ adenovirus E1B 19-kDa interacting protein 3 like (BNIP3L), also known as Nix, are members of the BH3-only subfamily of Bcl-2 family proteins, and are critical for the induction of mitochondrial autophagy, in some instances, promotes survival by reducing radical oxygen species (ROS) and DNA damage under hypoxic conditions $(36,37)$. BNIP3 can also induce cell death; however, evidence is not conclusive whether increased BNIP3 expression alone is sufficient for an apoptotic response (37). However, a previous study indicated that (38) autophagic cell death is induced in cancer cell lines with functioning apoptotic mechanisms, hypothesizing that early induction of BNIP3 may be a protective response with prolonged induction leading to cell death.

Recent studies have identified that BNIP3 and BNIP3L, target genes of HIF-1 $\alpha$, are important in autophagy (39-40). BNIP3 serves a role in the induction of hypoxia-induced mitochondrial autophagy $(39,41,42)$. Autophagy can cause cell survival or death, and its role in IRI appears to be model- and/or tissue-dependent $(16,21)$. The present study demonstrated that BNIP3 and BNIP3 L were overexpressed following LIRI, and that preconditioning with dexmedetomidine effectively attenuated IRI by downregulating BNIP3 and BNIP3L. BNIP3 and BNIP3L are known to be target genes of HIF1- $\alpha$; however, in the present study preconditioning with dexmedetomidine upregulated the expression of HIF1- $\alpha$. The trend was not consistent with the downregulation of BNIP3 and BNIP3L that was observed following dexmedetomidine preconditioning, thus the exact mechanism underlying this effect remains unclear. 
In conclusion, the present study demonstrated that pulmonary IRI was associated with the upregulation of apoptosis and autophagy. Furthermore, preconditioning with dexmedetomidine provided protection against LIRI in a dose-dependent manner, partially through inhibiting autophagy. Dexmedetomidine was observed to upregulate HIF-1 $\alpha$ and downregulate BNIP3 and BNIP3 L in the LIRI model in the current study. The results of present study highlight a potential clinical application for dexmedetomidine in reducing LIRI.

\section{Acknowledgements}

The present study was supported by the National Natural Science Fund (grant no. U1404807) and Medical Science Research Project of Henan Province (grant no. 201602227).

\section{References}

1. Christie JD, Carby M, Bag R, Corris P, Hertz M and Weill D ISHLT Working Group on Primary Lung Graft Dysfunction: Report of the ISHLT Working Group on Primary Lung Graft Dysfunction part II: Definition. A consensus statement of the International Society for Heart and Lung Transplantation. J Heart Lung Transplant 24: 1454-1459, 2005.

2. Ng CS, Wan S, Yim AP and Arifi AA: Pulmonary dysfunction after cardiac surgery. Chest 121: 1269-1277, 2002.

3. Shimamoto A, Pohlman TH, Shomura S, Tarukawa T, Takao M and Shimpo H: Toll-like receptor 4 mediates lung ischemia-reperfusion injury. Ann Thorac Surg 82: 2017-2023, 2006.

4. Ambrosio G and Tritto I: Reperfusion injury: Experimental evidence and clinical implications. Am Heart J 138: S69-S75, 1999.

5. Reino DC, Pisarenko V, Palange D, Doucet D, Bonitz RP, Lu Q, Colorado I, Sheth SU, Chandler B, Kannan KB, et al: Trauma Hemorrhagic Shock-Induced Lung Injury Involves a Gut-Lymph-Induced TLR4 Pathway in Mice. PLoS One 6: e14829, 2011

6. Jiang L, Li L, Shen J, Qi Z and Guo L: Effect of dexmedetomidine on lung ischemia-reperfusion injury. Mol Med Rep 9: 419-426, 2014.

7. Shen J, Fu G, Jiang L, Xu J, Li L and Fu G: Effect of dexmedetomidine pretreatment on lung injury following intestinal ischemia-reperfusion. Exp Ther Med 6: 1359-1364, 2013.

8. Ogata M, Hino S, Saito A, Morikawa K, Kondo S, Kanemoto S, Murakami T, Taniguchi M, Tanii I, Yoshinaga K, et al: Autophagy is activated for cell survival after endoplasmic reticulum stress. Mol Cell Biol 26: 9220-9231, 2006.

9. Tra T, Gong L, Kao LP, Li XL, Grandela C, Devenish RJ, Wolvetang E and Prescott M: Autophagy in human embryonic stem cells. PLoS One 6: e27485, 2011.

10. Kuma A, Hatano M, Matsui M, Yamamoto A, Nakaya H, Yoshimori T, Ohsumi Y, Tokuhisa T and Mizushima N: The role of autophagy during the early neonatal starvation period. Nature 432: 1032-1036, 2004.

11. Komatsu M, Waguri S, Ueno T, Iwata J, Murata S, Tanida I, Ezaki J, Mizushima N, Ohsumi Y, Uchiyama Y, et al: Impairment of starvation-induced and constitutive autophagy in Atg7-deficient mice. J Cell Biol 169: 425-434, 2005.

12. Suzuki C, Isaka Y, Takabatake Y, Tanaka H, Koike M, Shibata M, Uchiyama Y, Takahara S and Imai E: Participation of autophagy in renal ischemia/reperfusion injury. Biochem Biophys Res Commun 368: 100-106, 2008.

13. Jiang M, Liu K, Luo J and Dong Z: Autophagy is a renoprotective mechanism during in vitro hypoxia and in vivo ischemia-reperfusion injury. Am J Pathol 176: 1181-1192, 2010.

14. Gump JM, Staskiewicz L, Morgan MJ, Bamberg A, Riches DW and Thorburn A: Autophagy variation within a cell population determines cell fate through selective degradationof Fap-1. Nat Cell Biol 16: 47-54, 2014.

15. Zhang D, Li C, Zhou J, Song Y, Fang X, Ou J, Li J and Bai C: Autophagy protects against ischemia/reperfusion-induced lung injury through alleviating blood-air barrier damage. J Heart Lung Transplant 34: 746-755, 2015.
16. Zhang J, Wang JS, Zheng ZK, Tang J, Fan K, Guo H and Wang JJ: Participation of autophagy in lung ischemia-reperfusion injury in vivo. J Surg Res 182: e79-e87, 2013.

17. Luce JM: Acute lung injury and the acute respiratory distress syndrome. Crit Care Med 26: 369-376, 1998.

18. Zhang XY, Liu ZM, Wen SH, Li YS, Li Y, Yao X, Huang WQ and Liu KX: Dexmedetomidine administration before, but not after, ischemia attenuates intestinal injury induced by intestinal ischemia-reperfusion in rats. Anesthesiology 116: 1035-1046, 2012.

19. Lempiäinen J, Finchenberg P, Mervaala EE, Storvik M, Kaivola J, Lindstedt K, Levijoki J and Mervaala EM: Dexmedetomidine preconditioning ameliorates kidney ischemia-reperfusion injury. Pharmacol Res Perspect 2: e00045, 2014.

20. de Perrot M, Liu M, Waddell TK and Keshavjee S: Ischemia-reperfusion-induced lung injury. Am J Respir Crit Care Med 167: 490-511, 2003.

21. Zimmerman BJ and Granger DN: Mechanisms of reperfusion injury. Am J Med Sci 307: 284-292, 1994.

22. De Greef KE, Ysebaert DK, Ghielli M, Vercauteren S, Nouwen EJ, Eyskens EJ and De Broe ME: Neutrophilsand acute ischemia-reperfusion injury. J Nephrol 11: 110-122, 1998.

23. Oredsson S, Plate G and Qvarfordt P: Experimental evaluation of oxygen free radical scavengers in the prevention of reperfusion injury in skeletal muscle. Eur J Surg 160: 97-103, 1994.

24. Deby C and Goutier R: New perspectives on the biochemistry of superoxide anion and the efficiency of superoxide dismutases. Biochem Pharmacol 39: 399-405, 1990.

25. Feinman R, Deitch EA, Watkins AC, Abungu B, Colorado I, Kannan KB, Sheth SU, Caputo FJ, Lu Q, Ramanathan M, et al: HIF-1 mediates pathogenic inflammatory responses to intestinal ischemia-reperfusion injury. Am J Physiol Gastrointest Liver Physiol 299: G833-G843, 2010

26. Zhao X, Jin Y, Li H, Wang Z, Zhang W and Feng C: Hypoxia-inducible factor 1 alpha contributes to pulmonary vascular dysfunction in lung ischemia-reperfusion injury. Int J Clin Exp Pathol 7: 3081-3088, 2014.

27. Scherz-Shouval R, Shvets E, Fass E, Shorer H, Gil L and Elazar Z: Reactive oxygen species are essential for autophagy and specifically regulate the activity of Atg4. EMBOJ 26: 1749-1760, 2007.

28. Høyer-Hansen M, Bastholm L, Szyniarowski P, Campanella M, Szabadkai G, Farkas T, Bianchi K, Fehrenbacher N, Elling F, Rizzuto R, et al: Control of macroautophagy by calcium, calmodulin-dependent kinase kinase-beta, and Bcl-2. Mol Cell 25: 193-205, 2007.

29. Zhang J, Wang JS, Zheng ZK, Tang J, Fan K, Guo H and Wang JJ: Participation of autophagy in lung ischemia-reperfusion injury in vivo. J Surg Res 182: e79-e87, 2013.

30. Matsui Y, Kyoi S, Takagi H, Hsu CP, Hariharan N, Ago T, Vatner SF and Sadoshima J: Molecular mechanisms and physiological significance of autophagy during myocardial ischemia and reperfusion. Autophagy 4: 409-415, 2008.

31. Zhang D, Li C, Zhou J, Song Y, Fang X, Ou J, Li J and Bai C: Autophagy protects against ischemia/reperfusion-induced lung injury through alleviating blood-air barrier damage. J Heart Lung Transplant 34: 746-755, 2015.

32. Maiuri MC, Zalckvar E, Kimchi A and Kroemer G: Selfeating and self-killing: Crosstalk between autophagy and apoptosis. Nat Rev Mol Cell Biol 8: 741-752, 2007.

33. Eisenberg-Lerner A, Bialik S, Simon HU and Kimchi A: Life and death partners: Apoptosis, autophagy and the cross-talk between them. Cell Death Differ 16: 966-975, 2009.

34. Feng Z, Zhang H, Levine AJ and Jin S: The coordinate regulation of the 553 and mTOR pathways in cells. Proc Natl Acad Sci USA 102: 8204-8209, 2005.

35. Kim SY, Song X, Zhang L, Bartlett DL and Lee YJ: Role of Bcl-xL/Beclin-1 in interplay between apoptosis and autophagy in oxaliplatin and bortezomib-induced cell death. Biochem Pharmacol 88: 178-188, 2014.

36. Hamacher-Brady A, Brady NR, Logue SE, Sayen MR, Jinno M, Kirshenbaum LA, Gottlieb RA and Gustafsson AB: Response to myocardial ischemia/reperfusion injury involves Bnip3 and autophagy. Cell Death Differ 14: 146-157, 2007.

37. Tracy K, Dibling BC, Spike BT, Knabb JR, Schumacker P and Macleod KF: BNIP3 is an RB/E2F target gene required for hypoxia-induced autophagy. Mol Cell Biol 27: 6229-6242, 2007. 
38. Azad MB, Chen Y, Henson ES, Cizeau J, McMillan-Ward E, Israels SJ and Gibson SB: Hypoxia induces autophagic cell death in apoptosis-competent cells through a mechanism involving BNIP3. Autophagy 4: 195-204, 2008.

39. Zhang H, Bosch-Marcc M, Shimoda LA, Tan YS, Baek JH, Wesley JB, Gonzalez FJ and Semenza GL: Mitochondrial autophagy is an HIF-1-dependent adaptive metabolic response to hypoxia. J Biol Chem 283: 10892-10903, 2008

40. Novak I, Kirkin V, McEwan DG, Zhang J, Wild P, Rozenknop A, Rogov V, Löhr F, Popovic D, Occhipinti A, et al: Nix is selective autophagy receptor for mitochondrial clearance. EMBO Rep 11: 45-51, 2010.
41. Band M, Joel A, Hernandez A and Avivi A: Hypoxia-induced BNIP3 expression and mitophagy: In vivo comparision of the rat and the hypoxia-tolerant mole, Spalax ehrenbergi. FASEB J 23: 2327-2335, 2009.

42. Bellot G, Garcia-Medina R, Gounon P, Chiche J, Roux D, Pouysségur J and Mazure NM: Hypoxia-induced autophagy is mediated through hypoxia-inducible factor induction of BNIP3 and BNIP3L via their BH3 domains. Mol Cell Biol 29: 2570-2581, 2009. 